\title{
SPACE CHARGE EFFECT IN SECONDARY ELECTRON MONITORS
}

\author{
A.Tron, I.Merinov \\ MEPhI, Kashirskoe sh.31, 115409 Moscow, Russia
}

\section{Abstract}

Phase or spatial resolution of secondary electron monitors for, respectively, longitudinal or transverse bunch charge distribution measurements are restricted by the space charge effect of both a primary beam and secondary electron one. Simulation results of the effect in the monitors in an approach of ellipsoidal bunches of the primary beam with uniform charge density and taking into account the field of charges induced by the beam will be presented.

\section{INTRODUCTION}

Secondary electron monitors [1,2,3] for longitudinal bunch charge distribution measurement (BPD monitor) is the only tool at present for the measurement of short ion bunches with resolution up to $10 \mathrm{ps}$. This technique is the promising one for the bunch phase distribution monitoring of electron bunches with subpicosecond and far better resolution [4,5].

The space charge effect of the beam bunches is a main one restricting the monitor resolution $[6,7,8]$.

Using, as an example, the beam parameters of the specific ion linac at CERN (Linac 2), IHEP (URAL 60) and also the electron linac beam with the bunch population of $5 \cdot 10^{10}$ this effect is considered.

In the paper the same definitions and symbols as in $[2,4,5,6,8]$ are used.

In order to simplify the problem a two-dimensional charge distribution for the ellipsoidal ion bunches with uniform density and the circular cylinder with uniform charge distribution in the case of the electron beam have been taken. For these cases the expressions for the bunch field calculations were obtained on the base of the appropriate expressions published in $[9,10]$.

\section{GEOMETRY}

The following geometry of the primary converters of the considered secondary electron monitors, where the perturbation of the secondary electron is occurred, is shown in Fig.1.

The geometry shown in Fig.1a was used in the case of the ion beams, so named geometry 1 , and geometry 2 in Fig. 1b - for the case of the electron beam will be taken.
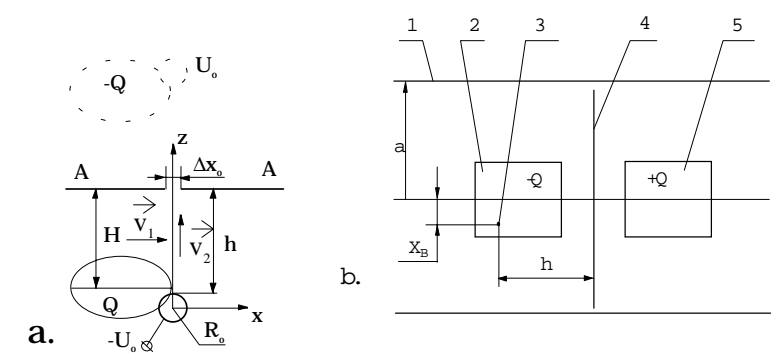

Figure 1: Geometry of primary converter with crossed (a) and coincident $(\mathrm{b})$ beams.

In Fig. 1a the ion beam with charge $\mathrm{Q}$ places from the conducting wall (A-A) of the monitor resonator on a distance $H$. The secondary electron emitter with the $\mathrm{R}$ radius being under negative voltage $\mathrm{U}_{\mathrm{o}}$ is apart of the wall on the h-distance. The ion bunch moves in the $\mathrm{x}$ direction. Charges induced by the beam on the wall was taken into account through its image.

In Fig.1b the bunch (2) and its image (5) move in the circular conducting cylinder (1) with the a-radius crossing the wire emitter (with radius $\mathrm{R}_{\mathrm{o}}$ ) being apart from the beam axis and the conducting wall (4) of the monitor resonator on $\mathrm{X}_{\mathrm{B}}$ and h distances respectively.

Everywhere below the following beam parameters are used: W - kinetic energy of the beam particle; $f$ frequency of the bunch accelerating field; I - the beam current; $\mathrm{N}$ - the bunch population; $\mathrm{R}$ - the beam radius; $\Delta \Phi$ - the bunch phase length on the f-frequency.

The perturbations of the secondary electron are described by the following parameters: $\Delta \varphi_{\mathrm{q}}$ - transit time spread (in unit of phase of the f-frequency) of the electrons reached the wall and started for a time of the bunch crossing by the emitter; $\delta q$ - electron relative momentum spread at the wall caused by the bunch space charge effect; $\Delta \mathrm{x}_{\mathrm{q}}^{\prime}$ - broadening of the electron beam divergence at the wall.

It should be noted that for reaching high resolution about $1^{\circ}$ and less the above mentioned parameters, namely: $\Delta \varphi_{\mathrm{q}}, \delta \mathrm{q}, \Delta \mathrm{x}_{\mathrm{q}}^{\prime}$ do not have to be in excess of $0.5^{\circ}$, $0.5 \ldots 1 \%, 10 \ldots 20 \mathrm{mrad}$ respectively.

\section{PROTON LINAC BEAM}

\subsection{URAL 60 Linac}

In Fig. 2 one can see the dependencies of $\Delta \varphi_{q}, \delta q, \Delta x_{q}^{\prime}$ vs. the emitter position relative to the beam axis for the beam 
of the URAL linac: $\mathrm{W}=62 \mathrm{MeV} ; \mathrm{I}=0.12 \mathrm{~A} ; \mathrm{R}=5 \mathrm{~mm}$; $\Delta \Phi=12^{\circ} ; \mathrm{f}=148.5 \mathrm{MHz}$, and for two emitters: the dotted lines - for $2 \mathrm{R}_{\mathrm{o}}=0.1 \mathrm{~mm} ; \mathrm{U}_{\mathrm{o}}=8 \mathrm{kV}$, and the solid line - for uniform emitter field. The H-distance has been fixed at $\mathrm{H}=15 \mathrm{~mm}$.

The behavior of the $\Delta \varphi_{\mathrm{q}}$-curves at different $\mathrm{R}_{\mathrm{o}}$ can be explained through Fig.3, where the $\mathrm{E}_{z}$-component of the bunch electric field strength vs. the z-coordinate is shown. If we take the point $\mathrm{A}$ as the electron start, then, taking into account that the more emitter electric field strength the more electron path for a time of the bunch crossing by the emitter, the electron will reach the point $\mathrm{B}$ or $\mathrm{C}$ for the appropriate magnitude of the $\mathrm{E}_{\mathrm{z}}$-strength. On the $A B$ path the sum effect of the bunch field is close to zero, and we have the another case for the AC path. In our case the AC path corresponds $2 \mathrm{R}_{\mathrm{o}}=0.1 \mathrm{~mm}$, and the $\mathrm{AB}$ path - for the emitter with large radius, i.e. when the emitter like a strip.

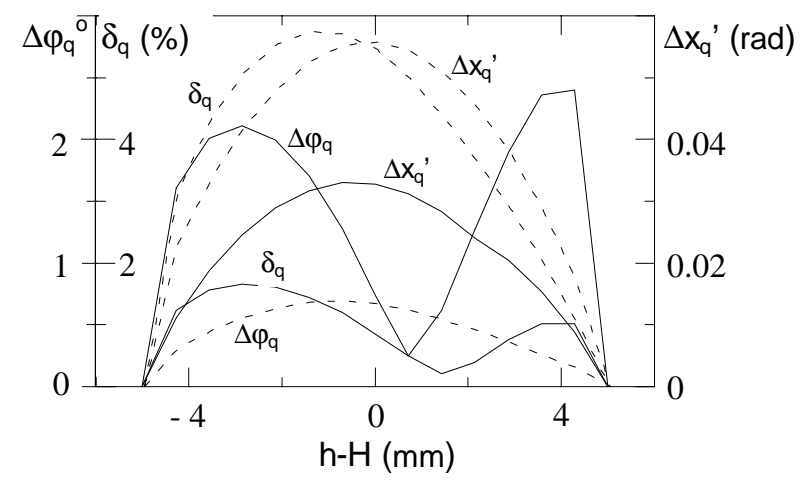

Figure 2: Space charge effect in the PC geometry 1 (see Fig.1a) for the URAL 60 linac beam vs. the emitter position.

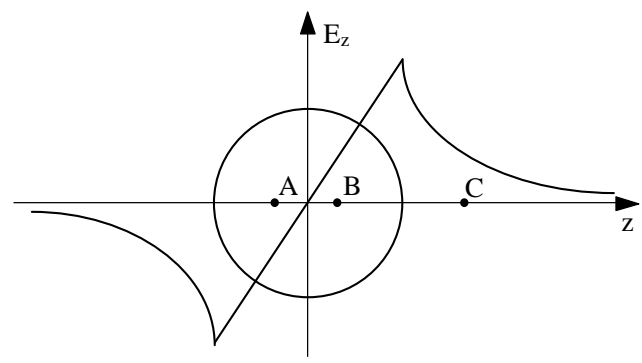

Figure 3: Electric field strength distribution.

In Fig.4 and Fig.5 the dependencies of the same parameters vs. the $\mathrm{U}_{\mathrm{o}}$-voltage or the bunch phase length $\left(2 \mathrm{R}_{\mathrm{o}}=0.1 \mathrm{~mm}\right)$ at the emitter position being coincident with the beam axis are shown respectively.

One can see the strong dependence of these parameters in the case of the small radius of the emitter.

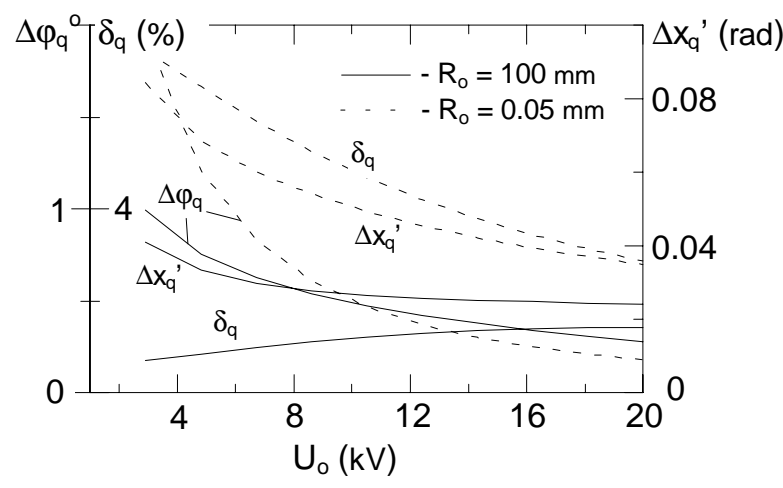

Figure 4: Space charge effect of the URAL 60 beam vs. the emitter voltage.

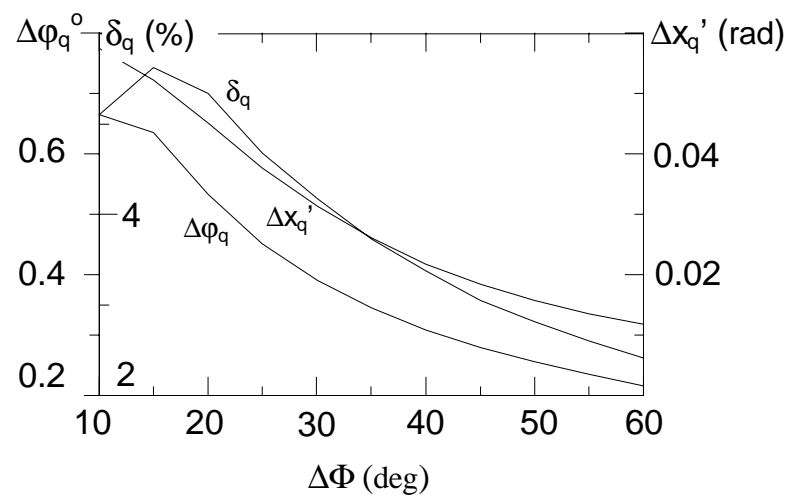

Figure 5: Space charge effect of the URAL 60 beam vs. its bunch length.

\subsection{CERN Linac 2}

Figure 6 represents the above mentioned dependencies for 3D-monitor [3] installed in the channel of the CERN Linac 2 for two proton energies, when the emitter of the $0.1 \mathrm{~mm}$ diameter under $10 \mathrm{kV}$ is used.

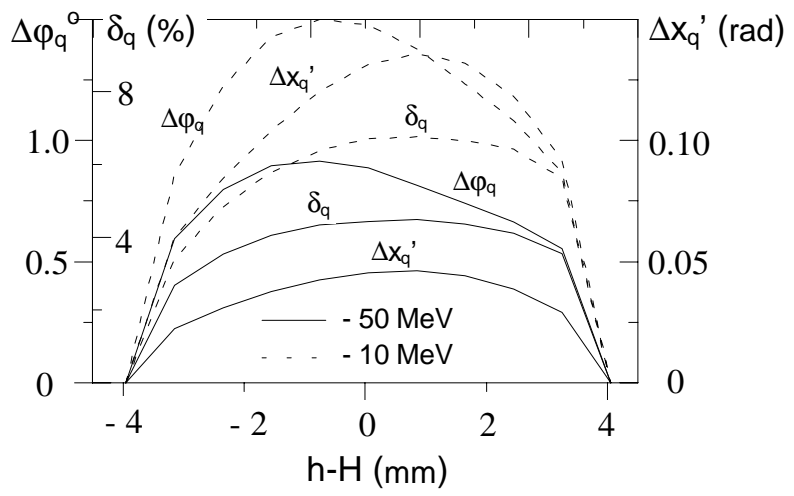

Figure 6: Space charge effect in the PC geometry 1 (see Fig.1a) for the CERN linac 2 beam vs. the emitter position.

The following beam parameters for these calculations have been taken: $\mathrm{I}=0.15 \mathrm{~A} ; \mathrm{f}=202 \mathrm{MHz}$; $\Delta \Phi=30^{\circ} ; \mathrm{R}=4 \mathrm{~mm}$. These dependencies have been 
obtained for the fixed h-distance of $20 \mathrm{~mm}$. Getting the high resolution at these parameters both the beam and the monitor is a problem.

In Fig.7 for the same case the perturbations in the vertical plane are shown, where: 1 - the beam cross section; 2 - the emitter; 3 - the plane of the y-collimator; 4 - the plane of multichannel collector. All sizes are given in $\mathrm{mm}$. Displacement of the electron in the collimator plane can reach about $0.8 \mathrm{~mm}$. Below, the curves of the equal electron displacement in $\mathrm{mm}$ (a) in the plane of the collimator and their additional divergence in mrad (b) at the same plane vs. the points of the electron start within the beam cross section are shown.
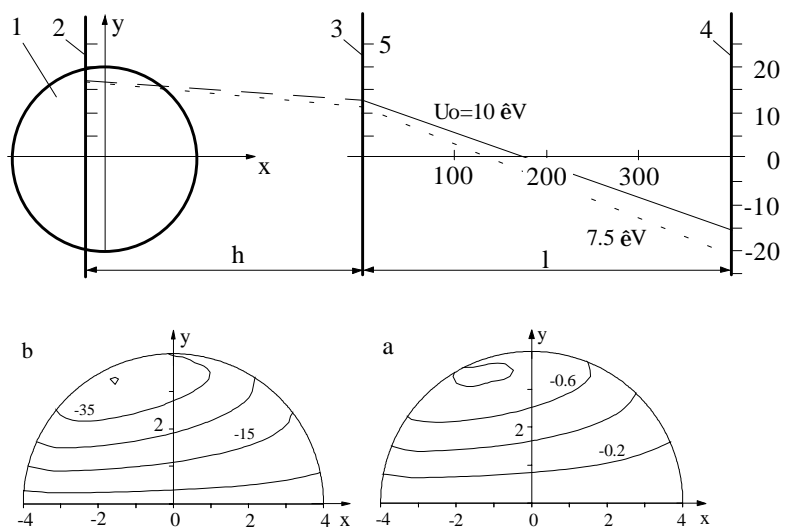

Figure 7: Electron trajectory perturbation in the 3Dmonitor (CERN linac 2) vs. initial secondary electron position.

\section{ELECTRON LINAC BEAM}

The perturbation of the secondary electrons in the monitor [11] with geometry 2 (see Fig.1b) caused by space charge effect of the electron beam are shown in Fig.8.

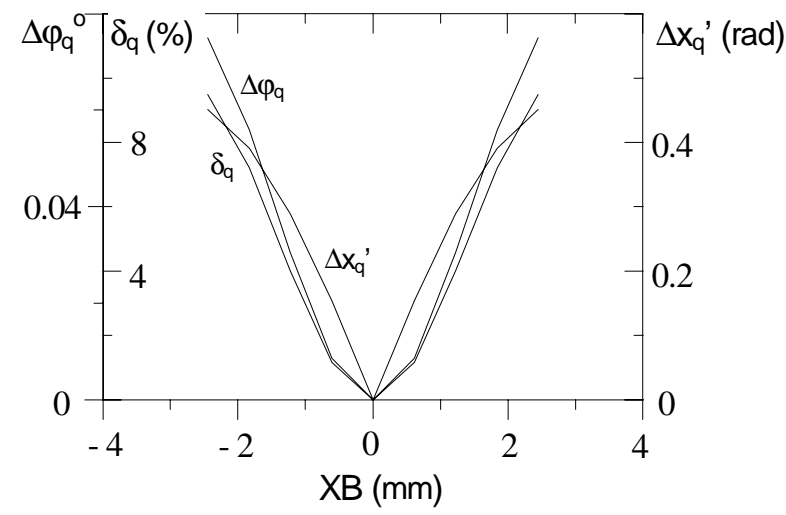

Figure 8: Space charge effect in the PC geometry 2 (see Fig.1b) vs. the emitter position.

The following beam parameters have been taken for these calculations: $\quad \mathrm{W}=500 \mathrm{MeV} ; \quad \mathrm{N}=5 \cdot 10^{10}$ el.; $\Delta \Phi=30^{\circ} ; \mathrm{f}=2.856 \mathrm{MHz} ; \mathrm{R}=2.5 \mathrm{~mm}$. The parameters of the monitor were: $R_{o}=0.025 \mathrm{~mm} ; U_{o}=4 \mathrm{kV}$; $\mathrm{h}=0.5 \mathrm{~mm}$.
From these dependencies one can conclude that the bunch phase distribution monitoring with resolution in subpicosecond range can be reached within the beam area of the $0.5 \mathrm{~mm}$ radius near the beam axis.

\section{CONCLUSIONS}

Represented dependencies of the secondary electron perturbations in the primary converter allow to determine the phase resolution of the monitor on the base of simple algorithm for the monitor resolution calculation represented in detail in the paper [6] both for the monitor with longitudinal and transverse rf-modulation of the secondaries, the definitions and symbols used there are the same as in this paper.

By choosing the optimal values of the monitor's parameters we can reach the high resolution for any rflinac beam, in particular, near the beam axis where for a rotationally symmetrical bunch its length and longitudinal emittance are maximum.

As to the 2D- beam current distribution measurement in the 3D-monitor [3] it should be noted that in the monitor there is no focusing in the y-direction, and the beam size measured in the y-direction (see Fig.7) will be smaller than it can be watched in the $\mathrm{x}$-direction up to $1 \mathrm{~mm}$ from both its sides. The alternative methods and devices have been proposed for the 2D- and 3Ddistribution measurements $[12,13.14]$ in the case of the considerable space charge effect where the focusing in each direction are used.

\section{ACKNOWLEDGMENTS}

The research was supported by the Russian Fund for Fundamental Investigations.

\section{REFERENCES}

[1] R.L.Witkover, NIM 137, 203 (1976).

[2] A.Tron, Proc. of the LINAC90 Conf., 477 (1990).

[3] S.K.Esin,et al., Proc. of the LINAC96 Conf.,193 (1996).

[4] A.Tron, et al., Proc. of the LINAC96 Conf., 514 (1996).

[5] A.Tron, et al., Proc. of the LINAC96 Conf., 517 (1996).

[6] A.Tron, Proc. of the DIPAC95 Conf., 60 (1995).

[7] A.Tron, V.Vorontsov, Proc. of the 10th All-Un. PAC, Dubna, 452 (1986).

[8] A.Tron, V.Shako, Proc. of the PAC93 Conf., 1216 (1993).

[9] R.S.Muratov,"Potentials of Allipsoid", Moscow, Atom Pub., 1976.

[10] W.R.Smythe,"Static and Dynamic Electricity", New York, 1950.

[11] A.Tron, I.Merinov, V.Smirnov, "Option of Method for Electron Bunch Phase Distribution Monitoring", this Conf.

[12] A.Tron, et al., Abstracts of the 12th All-Un. PAC, Moscow, 213 (1990).

[13] A.Tron, Proc. of the LINAC94 Conf., 917 (1994).

[14] A.Tron, AIP Conf. Proc. 333, 438 (1994). 